Treks. We started our VR working group in 2017 and included complementary therapist, psychologist, medical consultant, social worker, nurses, HCA's, IT support and creative therapist, ensuring a holistic approach to its application. Staff visited other hospices and VR conferences collating information. There was anxiety from staff about the content of what we were to show patients and how emotive that may be how would we vet the content before application without watching it all first? How would we screen patients? How would we collate feedback? How do we keep this safe for patients? etc. Contact was made with Greener Games - they gifted us 2 lightweight headsets, supported us with training and helped to develop a screening tool. A project lead and 2 IPADS were funded by the APDS grant.

Results Patients attending our day care sessions were offered a way to achieve life goals that they may not have thought possible because of their illness. A new tool is now available for nursing staff to help patients to relax and cope with anxieties and symptoms. Patients reported feeling physically calmer after VR sessions. Staff witnessed reduced respiratory rate in 5 out of 13 patients. No side effects of using VR were reported.

\section{UNDERSTANDING AND ACCESSIBILITY OF MENTAL HEALTH -COMPARING OUR UNMET NEEDS AS PART OF MULTI SITE AUDIT FOR SPECIALIST PALLIATIVE CARE SERVICES}

Emer McKenna, Rebecca Chubb, Sarah Yardley. Douglas Macmillan Hospice, North Staffordshire Combined Healthcare Trust, Central and North West London Trust

\subsection{6/spcare-2020-PCC.158}

Background Palliative care patients have significant mental health needs and there is no national standard for communitybased care when patients have significant mental health needs in context of unstable or progressive life limiting illness. Invitation to be part of multisite audit to compare findings in Central and North west London mirrored other areas nationally.

Aim To establish levels of need for liaison psychiatric services or alternative innovative services by conducting a baseline analysis of current needs in new and current referrals.

Method Agreement through clinical governance channels to partake in multi-site audit. Prospective audit over 3 month period. Assessment of whether these patients have suitable access to existing mental health services and specialist palliative care staff are able to engage these services in shared care. To determine the number of these patients who are open or have in the last one year been open to $\mathrm{MH}$ services.

Results Dementia patients were excluded if needs met by full hospice team including admiral nurse support. 22 patients identified over 3 month period through inpatient, day therapy MDT and complex discussions with community PCNS. 10 were new patients, 16 formal mental health diagnosis. Of the other 6-5 had been diagnosed with Dementia and had needed mental health support including being under section 3. Main diagnosis was depression and or anxiety. Majority treated with SSRI and Benzodiazepines. 1/3rd had ongoing CPN input.

Discussion $9 \%$ of our current referrals are for dementia. We have 2 Admiral nurses at our hospice who are able to mange this population making referral to traditional $\mathrm{MH}$ services less likely.
We are working collaboratively with local mental health trust identification of unmet need and recommendations for raising staff awareness and for a shared care pathway as part of a joint Quality improvement project.

\section{9 GLUCOSE MONITORING AND PREVENTION OF ADRENAL CRISIS IN PATIENTS PRESCRIBED HIGH-DOSE STEROIDS IN A SPECIALIST PALLIATIVE CARE UNIT AND SPECIALIST ONCOLOGY UNIT}

Erin Mercer, Timothy George, Eleanor M Smith. Sheffield Teaching Hospitals NHS Foundation Trust

\subsection{6/spcare-2020-PCC.159}

Background Corticosteroids are frequently prescribed in Palliative Care to improve symptoms from various conditions. However, they can affect glucose homeostasis and may cause steroid-induced adrenal insufficiency if patients become acutely unwell. Anecdotally, practice within hospital settings is varied.

Aims To audit prescriptions and management against Trust guidelines and then identify areas for improvement.

Methods Patients prescribed corticosteroids $10 \mathrm{mg}$ prednisolone (or equivalent) for 5 days in Weston Park Hospital (WPH) and Macmillan Palliative Care Unit (MPCU) were included. Data was collected from notes and Trust IT systems (Lorenzo and ICE) between 1st - 31st March, 2019. Findings were recorded using Survey Monkey.

Results Thirty patients were identified at WPH; eight at MPCU. Doses were documented $(100 \%)$ at both sites. Indications were less clear; $70 \%$ had documented indications at WPH $(n=21)$ vs $25 \%$ at MPCU $(n=2)$. Fewer had documented duration of therapy; $20 \%$ at WPH $(n=6)$ vs $0 \%$ at MPCU.

$100 \%$ of patients at MPCU had blood glucose measured daily $(n=8)$ vs $20 \%$ at WPH $(n=6) .80 \%$ identified as entering their last phase of life had steroids reviewed at MPCU $(n=4)$ vs $25 \%$ at WPH $(n=1) .100 \%$ who became acutely unwell had their steroid dose increased at MPCU $(n=2)$ vs $0 \%$ at WPH. No patients discharged with steroids (21 patients at WPH and one at MPCU) were given 'sick-day' education or rescue steroids. Only one patient at WPH was discharged with glucose monitoring (4.8\%).

Conclusions Glucose monitoring in patients taking high-dose steroids is better at MPCU than WPH, possibly due to existing interventions triggering review on consultant-led rounds; we hope to replicate this at WPH. Outpatient glucose monitoring and 'sick-day' education were universally poor, placing patients at risk of unmonitored diabetes and adrenal crisis. Further education is planned from Trust Diabetic and Endocrinology teams with a re-audit in 2020 .

\section{IMPROVING COLLABORATION BETWEEN RESPIRATORY AND PALLIATIVE MEDICINE, TO ADDRESS THE UNMET NEEDS OF PATIENTS WITH CHRONIC OBSTRUCTIVE PULMONARY DISEASE (COPD) IN THE LAST YEAR OF LIFE}

Debra S Morris, Stephanie Stolberg, Christine Seddon, Rachael Burton. Salford Royal NHS Foundation Trust

\subsection{6/spcare-2020-PCC.160}

Background COPD guidelines recommend early access to palliative care together with optimal therapy for people with 
advanced disease. An acute respiratory ward and an innovative One Stop Palliative Care COPD clinic was used to test the theory that early palliative care intervention supports self-management, advance care planning and reduces avoidable hospital admissions.

Methods The introduction of a weekly Gold Standard Framework (GSF) meeting and the use of GSF prognostic indicators were initiated on a respiratory ward, to identify COPD patients in the last year of life. Patients were offered palliative care support, in hospital and community, to help them live well with the disease. A One Stop Palliative Care COPD clinic was introduced to promote self-management and advance care planning.

Results

- Hospital referrals have increased from 20\% (Aug18-Jan 19) to $62 \%$ (Feb 19-July 19)

- Community palliative care support has increased from $21 \%$ to $70 \%$

- Increase in documented advance care plans has increased from $46 \%$ to $90 \%$

- $77 \%$ of patients were identified and referred for inclusion on the GSF register

- Patients were empowered to live well with the disease

- Outcome measures have shown that COPD patients have a greater symptom burden than other patients referred to palliative care.

- The project can be replicated across all disease groups

Conclusion This model has validated the theory that early referral to palliative care services for COPD patients in the last year of life, enhances patient care. It encourages and promotes living well with the disease, advance care planning with the intention of reducing avoidable hospital admissions.

\section{RESPECT, FIND OUT WHAT IT MEANS TO ME- AN AUDIT OF EMERGENCY TREATMENT PLANS}

Matthew Murden, Roop Gill, Sinead Henderson. Ashford and St Peter's NHS Trust

\subsection{6/spcare-2020-PCC.161}

Introduction ReSPECT is a national initiative led by the Resuscitation Council(UK). It encourages doctors to work in partnership with patients to establish their wishes and thoughts on future treatments should they become unwell. Moving away from old DNAR forms requires a significant culture shift. We replaced these forms at Ashford \& St Peter's Hospital NHS Trust November 2018. In this audit we aimed to assess the success of the rollout of ReSPECT forms hospital-wide in the first 3 months, aiming for more than $98 \%$ of patients having a valid form.

Methods Retrospective notes audit. The first 10 patients discharged from each ward, including patients who died, were selected for an electronic notes review looking for a ReSPECT form or documented evidence that a ReSPECT form was filled during admission. The presence of a ReSPECT form and the extent to which it was correctly filled out were recorded in a spreadsheet.

Results 209 patient records were examined (100 Male, 109 Female) across a mix of surgical and medical wards, average age of 77 years. A total of 87 forms were reviewed, meaning
42\% had a ReSPECT form present. Personal/clinical details and summary of emergency care and treatment recommendations were completed $70 \%$ of the time. Areas for improvement include mental capacity documentation and section 3 patient personal preferences.

Conclusions Although uptake initially appears poor in retrospect our standard of $98 \%$ was likely too high, a $42 \%$ uptake is a significant achievement for the first 3 months. By identifying areas for improvement we have been able to put in place initiatives to target these specifically. We recognise the need to continue to facilitate a change in culture within our trust and promote shared decision making and 'the conversation' as a process not a one off. We will reaudit next year.

\section{ANALYSIS AND IMPACT OF NON MEDICAL PRESCRIBING INTERVENTIONS AT JOHN TAYLOR HOSPICE}

T Nayyar, S Begum, C Hirsch. John Taylor Hospice, University of Birmingham

\subsection{6/spcare-2020-PCC. 162}

Introduction The growth in non-medical prescribing (NMP) by nurses and pharmacists has been well documented across a range of medical specialities. Prescribing by allied health professionals in palliative care is a relatively recent phenomenon. Outcome data for community palliative care prescribing is poorly characterised, and evidence of impact remains unsubstantiated. The purpose of this study was to gauge self-reported outcomes of prescribing interventions by nurse and pharmacist prescribers for patients in a community setting.

Methods Prescribing data was collected between 1st May and 1st July 2019. Data included perceived reasons for prescription issue and the outcome of the intervention. Data was anonymised and analysed using MS Excel to quantify the classes of drugs prescribed, and identify prescribing patterns. A second phase involved an online survey of medical and NMPs at the hospice to explore their views on independent prescribing.

Results Data from 7 nurse and 2 pharmacist NMPs were analysed. In over one third (35\%) of prescribing events, prescriptions were for routine symptom control such as pain or nausea. Provision of anticipatory injectable medicines was the second most common reason for prescription issue. Forty eight percent of patients prescribed medicines for symptom control achieved faster access to treatment. In $57 \%$ of prescription events a GP home visit was prevented. Analysis of the surveys is pending and themes arising from these will be shared amongst the hospice prescribing team.

Conclusion As a result of non-medical prescribing, patients receiving community palliative care were able to access crucial medicines in a timely manner which may have facilitated better symptom control. The study's main limitation was that prescribers completed data collection forms themselves, which may have influenced their choice of outcome. Potential savings of approximately $£ 2,595$ in GP visits suggest that utilisation of NMP prescribing should be more widely considered in palliative care teams. 